\title{
Effects of leptin on the expression of fatty acid-binding proteins in human placental cell cultures
}

\author{
ATHANASIOS V. MOUSIOLIS ${ }^{1}$, PANAGOULA KOLLIA ${ }^{2}$, CHARA SKENTOU $^{1}$ and IOANNIS E. MESSINIS ${ }^{1}$ \\ Departments of ${ }^{1}$ Obstetrics and Gynaecology, and ${ }^{2}$ Cytogenetics and Molecular Genetics, \\ Medical School, University of Thessalia, University Hospital, Larissa, Greece
}

Received June 9, 2011; Accepted September 27, 2011

DOI: $10.3892 / \mathrm{mmr} .2011 .686$

\begin{abstract}
The present study aimed to evaluate the protein expression of the transmembrane translocase FAT/CD36 and cytoplasmic H-FABP and L-FABP in human trophoblast tissue and evaluate the effects of exogenous leptin upon differential expression of each biomolecule; consequently, it aimed to derive information regarding the effects of leptin upon the expression of proteins implicated in fatty acid metabolism. Protein and total RNA were isolated from 72 samples of trophoblast tissue obtained from chorionic villous sampling. Of these, 36 samples were evaluated for protein (supernatant and pellet fraction separated) and the other 36 for total RNA expression. For each subgroup of samples, 12 were treated immediately and 24 were cultured. Half of the cultured samples were treated with $10 \mathrm{ng} / \mathrm{ml}$ exogenously added leptin and the other half were untreated. Western blotting and PCR techniques were used for the evaluation of biomolecule expression. Our results were obtained from samples at a mean gestational week of $12^{+5}\left(n=72\right.$; min, $11^{+0}$; max $14^{+1} \mathrm{gw}$; SD,0.89). In promptly treated samples we observed the presence of FAT/CD36 protein and absence of cytoplasmic FABPs. In the latter, only mRNA transcription of H-FABP was noted. A cytoplasmic pool of FAT/CD36 was also noted in the supernatant fraction of proteins. For cultured samples, when leptin was added, a statistically significant increase in FAT/CD36 protein expression was observed $(n=24, p<0.001$; mean difference, 0.219; SD, 0.0315; CI, -0.284 to -0.154). In our study we demonstrated the protein and mRNA expression of biomolecules implicated in fatty acid metabolism in human placenta. A cytoplasmic pool of the transmembrane protein FAT/CD36 was noted. Leptin caused the increase in FAT/ CD36 protein expression in the cultured samples. Therefore, we conclude that leptin has an immediate effect and plays a role in lipid metabolism in human placenta.
\end{abstract}

Correspondence to: Mr. Athanasios V. Mousiolis, 44 Papadiamantopoulou Street, Ilissia 15771, Athens, Greece

E-mail: thanasism@yahoo.com

Key words: leptin, FAT/CD36, H-FABP, L-FABP, human placenta

\section{Introduction}

Long chain fatty acids (LCFAs) are taken up into cells by passive diffusion and by protein-mediated mechanisms involving a number of fatty acid-binding proteins $(1,2)$. For the growing embryo, LCFA uptake is crucial for normal nervous system and organ development. Fatty acids are required by the developing fetus to maintain the fluidity, permeability and conformation of membranes, as precursors of important bioactive compounds such as the prostacyclins, prostaglandins, thromboxanes and leukotrienes and as a source of energy (3). The uptake of fatty acids from the maternal circulation to the embryo is mediated through the placenta. Differences in LCFA concentrations between maternal and fetal circulation, the latter being higher, lead to the conclusion that active mechanisms play a significant role in this divergence.

It has been shown from studies of muscle and heart tissue that FAT/CD36 plays a critical role in lipid transport via mechanisms yet to be fully elucidated. Acute and chronic stimuli have been shown to exert contradictory results on FAT/CD36 expression and thus in lipid uptake $(4,5)$. Furthermore, the precise cooperation and coordination mechanism among cytosolic (mainly H-FABP and L-FABP) and membrane (FABPpm, FATP and FAT/CD36) fatty acid-binding proteins requires additional elucidation. Little work has been carried out to clarify the role that these fatty acid-binding proteins in LCFA transportation through the placenta. The majority of studies have been performed on experimental models such as the BeWo human choriocarcinoma cell line, which is not capable of precisely representing the normal human trophoblast tissue profile.

Additionally, to our knowledge there have not been any studies on the role of cytotrophoblast cells or the effect that other substances and hormones have on cytotrophoblast cell behavior. Leptin in particular is thought to be implicated in various aspects of placental lipid uptake regulation and has not been studied conclusively to date.

The present study aimed to investigate the expression of cytosolic fatty acid-binding proteins (H-FABP and L-FABP) and transmembrane fatty acid translocase/CD36 protein (FAT/ CD36) in newly obtained trophoblastic tissue and in cytotrophoblast cell cultures. The effect of leptin on the expression of these proteins was also studied. 


\section{Materials and methods}

Trophoblastic tissue was obtained from women undergoing chorionic villous sampling for the purpose of diagnosing chromosomal abnormalities. The study was approved by the Ethics Committee of the University of Thessaly. Informed consent was obtained from each participating subject. Samples used for the experiments were those of normal karyotypes.

In total, 72 samples were collected and used in the study. Due to the small quantity of trophoblast tissue in each sample, they were randomly selected either for protein or RNA study. Thereafter, 36 specimens were evaluated for protein (supernatant and pellet fraction separated) and the other 36 for total RNA expression. For each subgroup of samples, 12 were treated immediately and 24 were cultured. Half of the cultured samples were treated with exogenous added leptin and the other half were untreated.

Trophoblast tissue culture. Biopsy tissue was treated promptly to prevent degradation of tissue. Briefly, obtained tissue was thoroughly and repeatedly washed with PBS solution to remove any blood clots present. The washed pellet was incubated for $40 \mathrm{~min}$ in enzymatic dissociation solution containing $1 \mathrm{mg} / \mathrm{ml}$ collagenase and $1 \mathrm{mg} / \mathrm{ml}$ trypsin. Subsequently, the enzymatic solution was neutralized by adding an excess volume of culture medium. Cells were mechanically dissociated to a further extent and then centrifuged. The supernatant was discarded and the pellet was resuspended in Chang-D (T105, Irvine Scientific, Santa Ana, CA, USA), a medium specifically optimized for culturing chorionic villi samples (CVSs) during prenatal care. Cells were transferred to $25 \mathrm{~cm}^{2}$ flasks (Sarstedt) and cultured at $37^{\circ} \mathrm{C}$ in a humidified incubator with a $5 \% \mathrm{CO}_{2}$ air atmosphere. Culture medium was changed at day 5 of culture. Culture duration was 10 days. Leptin at a final concentration of $10 \mathrm{ng} / \mathrm{ml}$ was added to half of the samples for the whole duration of culture. The samples to which leptin was added were selected randomly. Cells were collected and processed for the purposes of flow cytometry, biochemical analysis, total RNA and protein collection.

\section{Biochemical assays}

Flow cytometry. In order to determine the cell type population an anti-cytokeratin antibody was used. Briefly, 50-100 $\mu \mathrm{l}$ of cells was incubated for $15 \mathrm{~min}$ with cytokeratin clone RCK105 (Abcam, Cambridge, UK), and CD45 was used as a reference marker. For cytokeratin level estimation, DNA PrepReagent Kit (Beckman Coulter, Brea, CA, USA) and cytomics FC 500 Flow cytometer (Beckman Coulter) were used.

Additionally, $\beta$ chorionic gonadotropin ( $\beta$-hCG) secretion was measured in the culture medium supernatant of several samples on various days during culture. Briefly, $1 \mathrm{ml}$ of the supernatant was removed from the cultures, and $\beta$-hCG levels were measured using the appropriate ELISA kit (Elecsys and cobase analyzers).

Total RNA isolation and CDNA synthesis for qualitative PCR. Trophoblast tissue total RNA was isolated prior to and after 10 days of culture. Total cellular RNA was isolated using
Table I. Reagent mix and quantitative PCR conditions for cDNA synthesis.

\begin{tabular}{lrl}
\hline Reagents & Volume & PCR conditions \\
\hline Sample & $13.0 \mu 1$ & \\
$1^{\text {st }}$ strand buffer 5X & $4.0 \mu 1$ & $1.37^{\circ} \mathrm{C}$ for 60 min \\
MMLV & $1.0 \mu 1$ & $2.94^{\circ} \mathrm{C}$ for 2 min \\
dNTPs & $2.0 \mu 1$ & 3. Storage at $4^{\circ} \mathrm{C}$ \\
Total volume & $20.0 \mu 1$ & \\
\hline
\end{tabular}

the guanidinium isothiocyanate method. For quantitative PCR purposes, a volume equivalent to $1 \mu \mathrm{g}$ of total RNA of each sample was diluted with $1 \mu \mathrm{l}$ of random primer mix and $\mathrm{ddH}_{2} \mathrm{O}$ to a final volume of $13 \mu \mathrm{l}$ and incubated at $65^{\circ} \mathrm{C}$ for $10 \mathrm{~min}$. Cooling in ice for $5 \mathrm{~min}$ followed. The mix reagents and PCR conditions for the next step are shown in Table I. Retinoic acid receptor $\alpha$ sequences (RAR $\alpha$ ) were amplified in separate reactions as a control for the presence of amplifiable cDNA.

H-FABP and L-FABP gene probing. cDNA sequences corresponding to the H-FABP and L-FABP mRNA transcript were amplified with the appropriate primers (TIB Molbiol, Berlin, Germany) shown in Table II. PCR was performed in a $100-\mu 1$ reaction volume. Reagent mix for the two genes probed consisted of $5 \mu \mathrm{l}$ of sense and antisense primers, $10 \mu \mathrm{l}$ of 10X buffer mix, $5 \mu \mathrm{l}$ of $\mathrm{MgCl}_{2}, 2 \mu \mathrm{l}$ of dNTP mix and $0.4 \mu \mathrm{l}$ of Taq polymerase. PCR conditions are shown in Table III.

\section{Protein isolation}

Western blotting. For the purpose of protein study, proteins of cell cytoplasm and cellular membranes were isolated from cell extracts. Western blot analysis was performed to investigate the protein expression of cytoplasmic H-FABP and L-FABP proteins and transmembrane FAT/CD36 protein using the respective antibodies. For the L-FABP and FAT/CD36 studies, normal human hepatic cell extract was used as a positive control. Mouse heart cell extract (sc-2254; Santa Cruz Biotechnology, Santa Cruz, CA, USA) was used as positive control for H-FABP protein. Cells were washed three times with ice-cold PBS and lysed with NET-Triton Lysis Buffer (0.01 M Tris-Cl, $0.1 \mathrm{NaCl}, 1 \mathrm{mM}$ EDTA pH 7.4, $1 \%$ Triton X-100, $10 \%$ glycerol, $0.1 \%$ SDS, $0.5 \%$ sodium deoxycholate and a cocktail of protease inhibitors). Aliquots of lysates containing $40 \mu \mathrm{g}$ of total protein for FAT/CD36, H-FABP or L-FABP detection were run on 4-12\% NuPAGE Tris-Acetate gel (Invitrogen, Carlsbad, CA, USA) under denaturing and reducing conditions. Proteins were transferred to PVDF membranes (BioRad, USA). Nonspecific binding of the antibody to the membrane was blocked by a 1-h incubation with 5\% (w/v) non-fat dry milk/0.01 (v/v) Tween-20 in PBS.

Immunoblot analysis was performed using mouse monoclonal anti-FAT/CD36 (1:500 dilution, ab17044; Abcam) mouse monoclonal anti-H-FABP (1:100 dilution, ab16915; Abcam) and mouse monoclonal anti-L-FABP (1:100 dilution, ab17159; Abcam). Human F-actin monoclonal antibody (Serotec, Oxford, UK) was used as a protein marker for 
Table II. Sense and antisense primers sequences for H-FABP and L-FABP probing.

\begin{tabular}{lccl}
\hline Gene & Gene Bank accession no. & Product size & \multicolumn{1}{c}{ Sequence } \\
\hline H-FABP & NM_004102 & $138 \mathrm{bp}$ & $\begin{array}{l}\text { Sense: 5'-AGCATCACTATGGTGGACG-3' } \\
\text { Antisense: 5'-GATGATTGTGGTAGGCTTGG-3' }\end{array}$ \\
L-FABP & & Sense: 5'-CGCTGGGTCCAAAGTGAT-3' \\
& NM_001443 & Antisense: 5'-ATGTCGCCGTTGAGTTCG-3' \\
\hline
\end{tabular}

Table III. PCR conditions for H-FABP and L-FABP probing.

\begin{tabular}{lcc}
\hline PCR step & Cycles & Conditions \\
\hline Step 1 & 1 & $94^{\circ} \mathrm{C}$ for $5 \mathrm{~min}$ \\
Step 2 & 35 & $94^{\circ} \mathrm{C}$ for $1 \mathrm{~min}$ \\
& & $55^{\circ} \mathrm{C}$ for $1 \mathrm{~min}$ \\
Step 3 & 1 & $72^{\circ} \mathrm{C}$ for $1 \mathrm{~min}$ \\
Storage & & $72^{\circ} \mathrm{C}$ for $10 \mathrm{~min}$ \\
\hline
\end{tabular}

the quantification of the protein bands. Membranes were then immersed in ECL detection solution (Santa Cruz Biotechnology) and exposed to XAR-5 film (Kodak, Rochester, NY, USA) for autoradiography. Protein bands were quantified using an Epson GT-8000 laser scanner. Image analysis of scanned films was performed using ImageJ v1.38 software package (National Institutes of Health, USA). For each sample, the ratio of the protein band intensity relative to its respective F-actin band intensity was calculated.

Statistical analysis. Statistical analysis was performed using SPSS v.15 statistical package for Windows. Kolmogorov-Smirnov normality test was used to study gestational ages and protein expression distribution. ANOVA was used to compare expression patterns between 'fresh' and cultured samples with or without the addition of leptin, followed by LSD post-hoc analysis. Confidence interval was set at $95 \%(\mathrm{p}=0.05)$. Accurate $\mathrm{p}$-scores were noted and presented when significantly less than 0.05 .

\section{Results}

Statistical analysis of the samples. CVS procedure was performed at an average gestational age of $12^{+5} \mathrm{gw}$ (gestational week) $\left(\mathrm{n}=72 ; \min , 11^{+0} ; \max , 14^{+1} \mathrm{gw} ; \mathrm{SD}, 0.89\right)$. The Kolmogorov-Smirnov test was applied to gestational age; normality could not be excluded ( $\mathrm{Z}=0.999, \mathrm{p}>0.05)$. Gestational week did not differ significantly between the subgroups.

$\beta$-chorionic gonadotropin $(\beta-h C G)$ and cytokeratin production. As proved by serial measurements at various days in the supernatant, $\beta$-hCG declined rapidly during the first days of culture but then remained relatively steady for the rest of the culture period (Fig. 1).

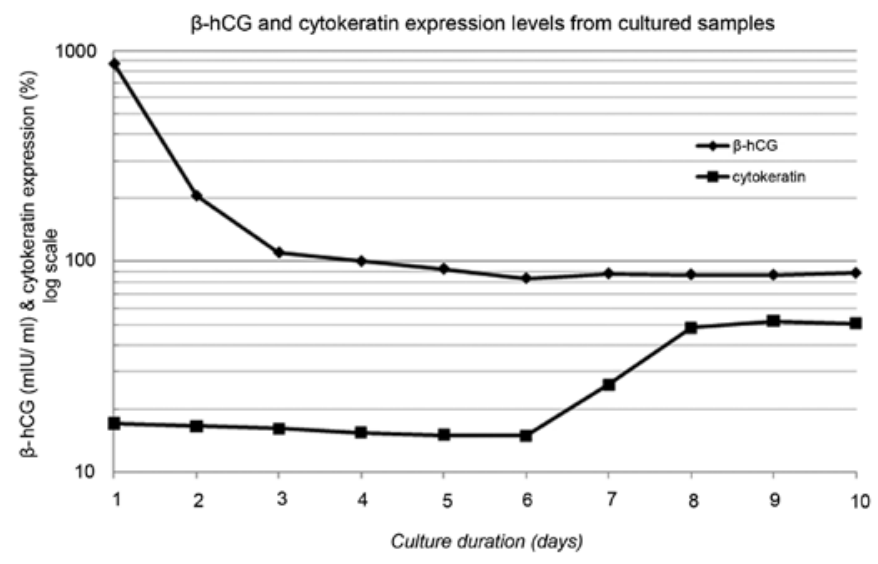

Figure 1. $\beta$-hCG secretion and cytokeratin expression from trophoblast tissue cultures on various days during culture.

In several samples examined for the presence of cytokeratin, fresh (untreated) samples proved to have an average percentage score of approximately 17\%. Later during culture, cytokeratin levels rose and remained steady, reaching $50 \%$ at the end of the 10-day culture period (Fig. 1). Results were negative for CD 45 in the cultured samples.

PCR H-FABP and L-FABP gene amplification. PCR analysis for L-FABP expression failed to show any mRNA transcripts present in samples examined regardless of the gestational week, culture duration and/or leptin presence in the culture medium (Fig. 2). In contrast, H-FABP mRNA transcripts were constantly present in the examined samples. Intensity varied between samples, exhibiting a more intense band signal in newly obtained tissue (Fig. 2).

Western blotting for H-FABP, L-FABP and FAT/CD36 protein expression. Western blot analysis failed to reveal any H-FABP or L-FABP protein expression in newly obtained or cultured samples regardless of the culture duration and/or the leptin presence in the culture medium (Fig. 3). Notably, the evidence of negative H-FABP protein expression is in contrast with the presence of mRNA transcripts revealed in similar samples.

By contrast, Western blot analysis exhibited marked FAT/CD36 protein expression on newly obtained pellets (Fig. 4), reflecting the presence of FAT/CD36 on cell membranes. In newly obtained samples, FAT/CD36 was also found among cytoplasmic proteins, a result consistent with 

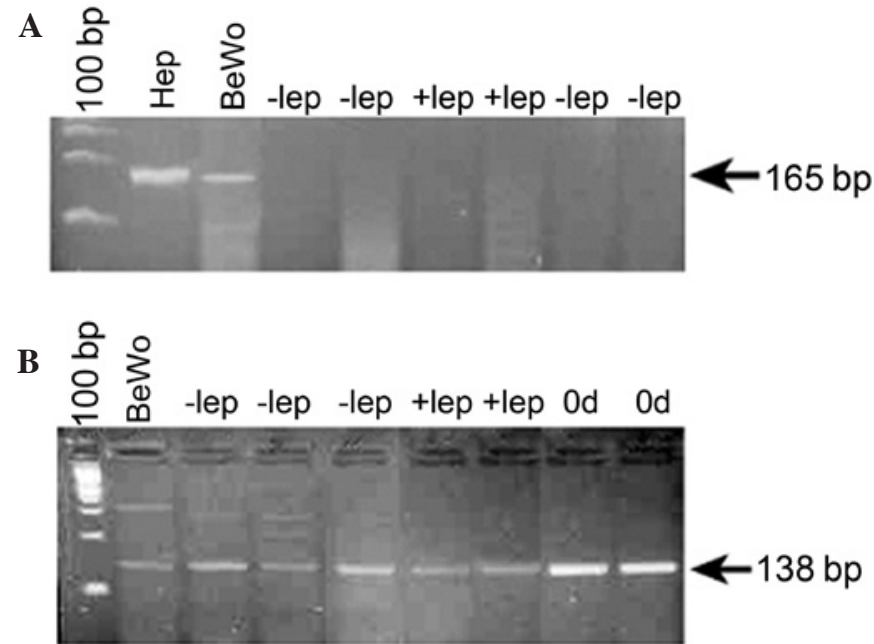

Figure 2. PCR analysis for L-FABP (A) and H-FABP (B) mRNA transcrip expression in newly obtained (0d) and cultured cells with (+lep) or without (-lep) added leptin. Hep, hepatic G2 neoplastic tissue extract; BeWo, human choriocarcinoma cell line.

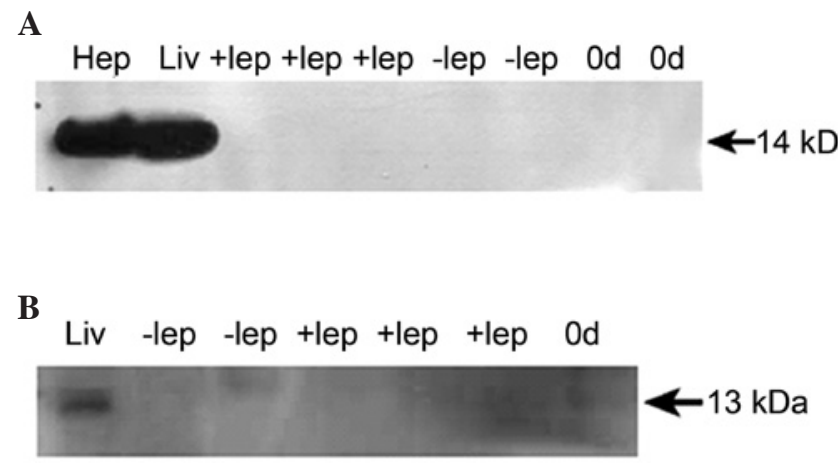

Figure 3. Western blot analysis for H-FABP (A) and L-FABP (B) in newly obtained (0d) samples and cultured samples with (+lep) or without (-lep) added leptin at a final concentration of $10 \mathrm{ng} / \mathrm{ml}$.

experimental works in other tissues showing the presence of a cytoplasmic FAT/CD36 pool (Fig. 5).

FAT/CD36 protein expression was also observed in cell membranes of cultured samples. There was a significant difference in expression between fresh and cultured samples. Fig. 4 shows FAT/CD36 protein expression in samples cultured for 10 days with or without $10 \mathrm{ng} / \mathrm{ml}$ of leptin present in the culture medium throughout the culture period. The difference in expression of FAT/CD36 in the cell membranes between newly obtained and long-term cultured samples with or without $10 \mathrm{ng} / \mathrm{ml}$ of leptin present in the culture medium is also shown (Fig. 6).

Statistical analysis showed statistically significant differences in the FAT/CD36 levels of the studied groups. Samples processed promptly showed higher levels of the protein probed than the cultured samples, even when exogenous leptin was added to the culture medium. However, leptin was found to augment the protein expression levels of FAT/CD36 in the cultured samples when compared with the untreated specimens. The mean expression of FAT/CD36 in the cultured

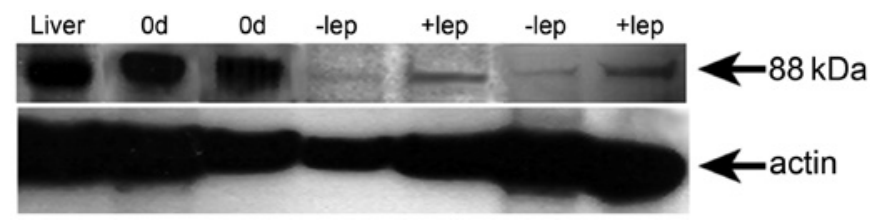

Figure 4. Western blot analysis on FAT/CD36 protein expression from protein pellet of newly obtained (0d) and cultured samples. +lep, samples with added leptin at $10 \mathrm{ng} / \mathrm{ml}$ final concentration; -lep, cultured samples with no added leptin. Liver, normal human hepatic cells protein extract.

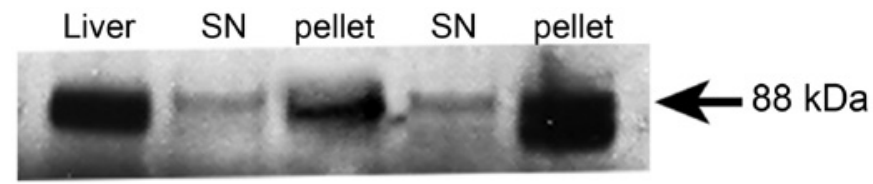

Figure 5. Western Blot analysis on FAT/CD36 protein expression from extracted pellet and supernatant (SN) of newly obtained samples (0d). Liver, normal human hepatic cell protein extract.

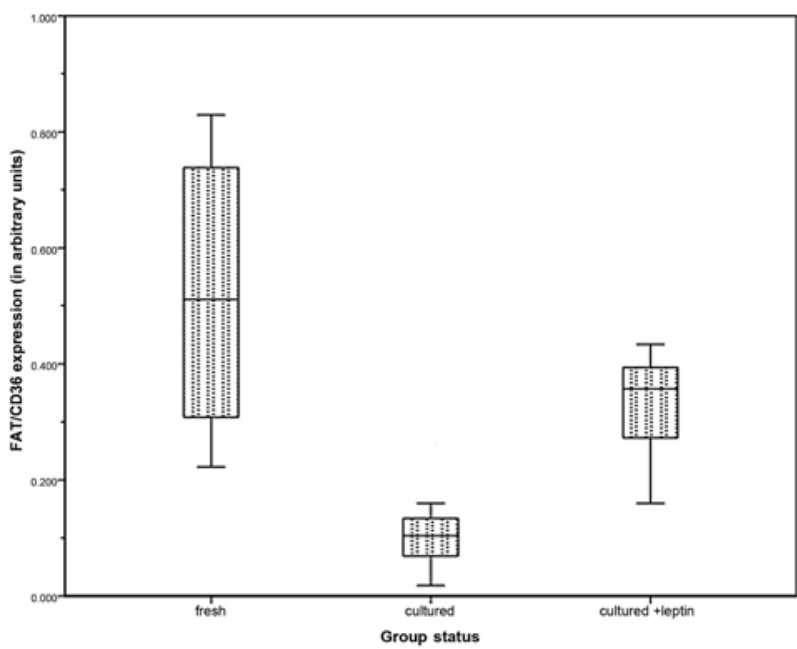

Figure 6. Expression difference between freshly isolated (fresh) and cultured samples with (cultured/+leptin) or without (cultured) the presence of exogenous added leptin. Using ANOVA and LSD post hoc statistical tests, a statistically significant difference was shown $(\mathrm{p}<0.001)$. In treated samples, leptin was added at a final concentration of $10 \mathrm{ng} / \mathrm{ml}$. There were 12 samples in each subgroup. The bottom of the boxplot represents the 25th percentile of values, and the top represents 75 th percentile. The line inside the box is the median value; upper whisker represents maximum and lower whisker the minimum value.

samples with leptin added was 0.329 , whereas the mean expression was 0.110 in samples cultured without the addition of leptin $(n=24 ; p<0.001$; mean difference, 0.219; SD, 0.0315; CI, -0.284 to -0.154 ) (Fig. 6).

\section{Discussion}

The majority of the studies published to date regarding the topic of our research come either from cell lines or from short-term cultures of trophoblast tissue obtained from term placentae that are not abundant in cytotrophoblast cells. Data 
from trophoblast cultures matching the gestational age of our study are, to our knowledge, very scarce. Our specimens came from late first- to early second-trimester normal gestations using CVS sampling. Other studies in early pregnancies, and the use of trophoblast tissue from CVS, are rarely encountered in the literature.

Although Kliman's Percoll gradient protocol of purification is routinely used in trophoblast cell cultures, when whole placentae were used, the amount of tissue collected using CVS did not permit the use of this protocol. Nevertheless, results obtained from our culture model are still comparable, since the cell population in each subgroup studied was similar and directly compared to its counterpart.

$\beta$ chorionic gonadotropin $(\beta-\mathrm{hCG})$ production is widely used as a marker of differentiation in trophoblast cell cultures and is thought to correlate positively with the differentiated syncytiotrophoblast presence. Syncytiotrophoblasts are considered to be the main site of $\beta$-hCG production and higher levels of this molecule are compatible with increased levels of syncytia in the cell population. Levels of $\beta$-hCG declined during the first days of culture but remained relatively steady afterwards. Cytokeratin levels rose and remained relatively high throughout the culture period. Cytokeratin levels also correlated with syncytiotrophoblast levels, with higher levels observed in cell populations rich in syncytia. The increase in cytokeratin levels further verified that trophoblast tissue was present. Additionally, cultures were inspected under microscopy under direct vision or crystal violet staining and evidence of syncytialization were noted and recorded (data not shown). Therefore, it is quite safe to speculate that trophoblast cells in the dish plate were viable, thus leading to a cell population comprising a high cytotrophoblast/syncytiotrophoblast fraction. This is in agreement with published data from other authors showing that syncytiotrophoblast cells lack adherence to the flask and are therefore readily flushed away during flask washing or culture medium renewal, whereas cytotrophoblasts are capable of adhering to the dish plate surface (6).

FAT/CD36 is a heavily glycosylated integral membrane protein with an apparent molecular mass of $88 \mathrm{kDa}$, which has been proposed to work as a transporter or as a translocase for fatty acids (7-9). FAT/CD36 is expressed abundantly in tissues active in fatty acid metabolism, such as heart, skeletal muscle, adipose tissue and intestine. Its expression profile corresponds with a role in fatty acid uptake, and its expression is modulated by conditions that alter lipid metabolism, such as diabetes mellitus and high-fat feeding. Studies show that FAT/CD36 is localized to both the microvillous and the basal membrane of human placenta (10). FAT/CD36 membrane localization permits the bidirectional flow of fatty acids in and out of the cells.

Altered FAT/CD36 expression is genetically linked with situations where insulin resistance and abnormal lipid metabolism occur in rats and humans $(11,12)$. In humans, FAT/CD36 is considered to be a significant factor in metabolic adaptation in diet and in susceptibility to certain abnormal situations related to diet $(4,13,14)$. For example, in situations where lipid uptake is increased, such as in pre-adipocyte to adipocyte differentiation, during perinatal and postnatal heart development, in the skeletal muscle of diabetic experimental animals and in the heart of animals under fat-rich diet (7). Insulin and leptin regulate the expression of proteins associated with fatty acid binding and transportation (namely FAT, FABPpm, FATP and cytosolic FABPs) in various tissues through PPAR (peroxisome proliferator-activated receptors) $(11,12,15)$.

Although there are no conclusive studies concerning the significance of FAT/CD36 in human placenta, in other tissues and organs its role is well established and considered to be crucial to the transportation of fatty acids through the cell membrane. For example, $60-80 \%$ of the fatty acid transportation in cardiomyocytes is facilitated through FAT/CD36. Similarly, FAT/CD36 plays a significant role in tissues such as adipose and muscle tissue and also in the intestine.

Various hypotheses exist regarding the precise mechanism of action of FAT/CD36 and the manner in which long chain fatty acid uptake is facilitated (16). Additionally, studies have revealed a possible interaction between FAT/CD36 and one or more proteins. One possible scenario is that of FAT/CD36 and cytosolic fatty acid-binding proteins (cFABPs), as shown in bovine mammary gland experiments. It was proposed that a complex of FAT/CD36 and cFABP exists in the mammary gland, that there is a proliferation regulatory mechanism or that the expression of the biomolecules depends on differentiation status of the cell (17). It has also been proposed that H-FABP is most likely to be a necessary partner for FAT/CD36 to facilitate fatty acid transport (3).

Our data show the abundant presence of FAT/CD36 protein expressed in trophoblast tissue taken from gestations around the beginning of the second trimester. Notably, our results are compatible with a cytoplasmic pool of FAT/CD36 in the syncytiotrophoblast cytosol. To our knowledge, this is the first time that such evidence has been revealed in syncytiotrophoblasts and is consistent with the cytosolic pool of FAT/CD36 found in other tissues, i.e. the muscle tissue (4). This evidence indicates a possible acute FAT/CD36 regulatory mechanism in syncytiotrophoblasts similar to that found in muscle tissue. In muscle tissue, cytosolic pool FAT/CD36 may be transported to the cell membrane in an acute mode after the stimulation of various factors, one of which is the administration of leptin $(4,11,12)$. Further research is required to elucidate the precise regulation of FAT/CD36 acute expression in syncytiotrophoblasts and whether it has a physiologic role in the lipid uptake mechanism.

In cultures, we revealed weak FAT/CD36 protein expression in the cell membrane. No cytosolic FAT/CD36 expression was noted. When leptin was administered, it caused an augmentation of FAT/CD36 protein expression. This is the first time, to our knowledge, that such evidence is reported for the human placenta. Thus, it is safe to speculate that the mode of action of leptin in the fatty acid transportation mechanism involves a direct effect on at least one of the biomolecules already assigned to this procedure.

The majority of authors conclude that the role of cytosolic FABP consists of being an intracellular carrier for fatty acids similar to albumin, thus allowing lipophilic fatty acids to be solubilized in the aqueous cytosolic environment. Additionally, a number of researchers have emphasized the role of cytoplasmic FABPs in removing fatty acids from the inner membrane and channelling them to their respective metabolic fates as a significant determinant of net uptake $(7,8)$. Regarding cytosolic FABPs, we observed no protein expression 
for H-FABP and L-FABP isoforms in newly obtained trophoblast tissue and trophoblast cell cultures. Other authors using self-made antibodies against heart- and liver-type FABP have revealed expression of those proteins in placentas within 5 to 30 weeks of gestation (18). The reason for this discrepancy in our specimens has yet to be elucidated.

Transcription of H-FABP and L-FABP was also examined. No L-FABP mRNA transcripts were observed in newly obtained or cultured samples. Notably, H-FABP mRNA transcripts were observed in newly obtained samples and trophoblast cultures, the former being more markedly expressed. This is in contrast to the absence of H-FABP protein expression in these cells. This finding cannot be fully interpreted but may indicate post-transcriptional regulatory mechanisms affecting protein expression. It may also be attributed to the characteristics of our working model or the conditions used for culturing the trophoblastic cells. Alternatively, it could indicate that H-FABP protein expression was below the sensitivity threshold of the method.

Freshly isolated trophoblast tissue exhibited a significantly higher level of FAT/CD36 when compared to trophoblast cells cultured with and without exogenous leptin added to the culture medium. This difference may be due to the increased presence, demonstrated by $\beta$-hCG production, of syncytiotrophoblasts in freshly isolated samples, since the syncytium layer plays a critical role in the transfer control regulatory mechanisms of all substances traveling from the maternal to fetal circulation, including fatty acids.

In conclusion, we examined the expression of certain proteins involved in fatty acid uptake in the human placenta. Our results show evidence of FAT/CD36 expression in syncytiotrophoblasts and cytotrophoblasts of mean 13 gestational weeks. Expression differences between these cell populations are significant and may be interpreted in respect to the physiological role syncytiotrophoblasts plays in the fatty acid uptake of the placenta. Every substance passing from maternal to fetal blood passes through the syncytiotrophoblast layer. Additionally, FAT/CD36 expression in the syncytiotrophoblast cytoplasm provides evidence, for the first time, of a cytoplasmic FAT/CD36 pool. The physiological role for this, if any, remains unclear; however, it may indicate a possible regulatory mechanism of FAT/CD36 distribution from cytoplasm to cellular membrane in an acute mode in placenta, consistent with similar evidence revealed previously in muscle tissue. Leptin affects FAT/CD36 expression in cytotrophoblast cells, providing evidence of a significant role in fatty acid transfer from maternal to fetal circulation and a direct involvement in the transportation mechanisms.

Additional studies towards a better clarification and understanding of the mechanisms underlying fatty acid uptake by the placenta are required. A valuable working model would be to detect whether leptin and its effect on FAT/CD36 also results in a net increase in fatty acid transfer in cultured trophoblastic tissue. Commercially available monoclonal antibodies for the other proteins involved in the process would also be of great benefit towards a clarification of this process, which is vital for the development of the fetus.

\section{References}

1. Hamilton JA and Kamp F: How are free fatty acids transported in membranes? Is it by proteins or by free diffusion through the lipids? Diabetes 48: 2255-2269, 1999.

2. Schaffer JE: Fatty acid transport: the roads taken. Am J Physiol Endocrinol Metab 282: E239-E246, 2002.

3. Haggarty P: Placental regulation of fatty acid delivery and its effect on fetal growth - a review. Placenta 23 (Suppl A): 28-38, 2002.

4. Bonen A, Luiken JJ, Arumugam Y, Glatz JF and Tandon NN: Acute regulation of fatty acid uptake involves the cellular redistribution of fatty acid translocase. J Biol Chem 275: 14501-14508, 2000.

5. Steinberg GR, Dyck DJ, Calles-Escandon J, et al: Chronic leptin administration decreases fatty acid uptake and fatty acid transporters in rat skeletal muscle. J Biol Chem 277: 8854-8860, 2002.

6. Guilbert LJ, Winkler-Lowen B, Sherburne R, Rote NS, Li H and Morrish DW: Preparation and functional characterization of villous cytotrophoblasts free of syncytial fragments. Placenta 23: 175-183, 2002.

7. Glatz JF and van der Vusse GJ: Cellular fatty acid-binding proteins: their function and physiological significance. Prog Lipid Res 35: 243-282, 1996.

8. Glatz JF, Luiken JJ, van Nieuwenhoven FA and van der Vusse GJ: Molecular mechanism of cellular uptake and intracellular translocation of fatty acids. Prostaglandins Leukot Essent Fatty Acids 57: 3-9, 1997.

9. Glatz JF, van Nieuwenhoven FA, Luiken JJ, Schaap FG and van der Vusse GJ: Role of membrane-associated and cytoplasmic fatty acid-binding proteins in cellular fatty acid metabolism. Prostaglandins Leukot Essent Fatty Acids 57: 373-378, 1997.

10. Campbell FM, Bush PG, Veerkamp JH and Dutta-Roy AK: Detection and cellular localization of plasma membraneassociated and cytoplasmic fatty acid-binding proteins in human placenta. Placenta 19: 409-415, 1998.

11. Chabowski A, Coort SL, Calles-Escandon J, et al: Insulin stimulates fatty acid transport by regulating expression of FAT/CD36 but not FABPpm. Am J Physiol Endocrinol Metab 287: E781-E789, 2004.

12. Glatz JF, Bonen A and Luiken JJ: Exercise and insulin increase muscle fatty acid uptake by recruiting putative fatty acid transporters to the sarcolemma. Curr Opin Clin Nutr Metab Care 5: 365-370, 2002.

13. Ibrahimi A, Bonen A, Blinn WD, et al: Muscle-specific overexpression of FAT/CD36 enhances fatty acid oxidation by contracting muscle, reduces plasma triglycerides and fatty acids, and increases plasma glucose and insulin. J Biol Chem 274: 26761-26766, 1999.

14. Zhang J, Phillips DI, Wang C and Byrne CD: Human skeletal muscle PPARalpha expression correlates with fat metabolism gene expression but not BMI or insulin sensitivity. Am J Physiol Endocrinol Metab 286: E168-E175, 2004.

15. Bonen A, Benton CR, Campbell SE, et al: Plasmalemmal fatty acid transport is regulated in heart and skeletal muscle by contraction, insulin and leptin, and in obesity and diabetes. Acta Physiol Scand 178: 347-356, 2003.

16. Abumrad N, Coburn C and Ibrahimi A: Membrane proteins implicated in long-chain fatty acid uptake by mammalian cells: CD36, FATP and FABPm. Biochim Biophys Acta 1441: 4-13, 1999.

17. Spitsberg VL, Matitashvili E and Gorewit RC: Association and coexpression of fatty-acid-binding protein and glycoprotein CD36 in the bovine mammary gland. Eur J Biochem 230: 872-878, 1995 .

18. Das T, Sa G and Mukherjea M: Characterization of cardiac fatty-acid-binding protein from human placenta. Comparison with placenta hepatic types. Eur J Biochem 211: 725-730, 1993. 